Electronic structure and luminescence mechanisms in $\mathrm{ZnMoO}_{4}$ crystals

This article has been downloaded from IOPscience. Please scroll down to see the full text article.

2011 J. Phys.: Condens. Matter 23365501

(http://iopscience.iop.org/0953-8984/23/36/365501)

View the table of contents for this issue, or go to the journal homepage for more

Download details:

IP Address: 131.169.39.151

The article was downloaded on 16/01/2012 at 12:47

Please note that terms and conditions apply. 


\title{
Electronic structure and luminescence mechanisms in $\mathrm{ZnMoO}_{4}$ crystals
}

\author{
D A Spassky ${ }^{1}$, A N Vasil'ev ${ }^{1}$, I A Kamenskikh ${ }^{2}$, V V Mikhailin ${ }^{1,2}$, \\ A E Savon ${ }^{2}$, Yu A Hizhnyi ${ }^{3}$, S G Nedilko $^{3}$ and P A Lykov ${ }^{4}$ \\ ${ }^{1}$ Skobeltsyn Institute of Nuclear Physics, M V Lomonosov Moscow State University, Leninskie Gory 1, \\ Building 2, 119991 Moscow, Russia \\ ${ }^{2}$ Physics Faculty, M V Lomonosov Moscow State University, Leninskie Gory 1, Building 2, \\ 119991 Moscow, Russia \\ ${ }^{3}$ Taras Shevchenko National University of Kyiv, Hlushkova avenue 4, 03680 Kyiv, Ukraine \\ ${ }^{4}$ A M Prokhorov General Physics Institute of RAS, Vavilov Street 38, 119991, Moscow, Russia \\ E-mail: deris2002@mail.ru
}

Received 8 June 2011, in final form 20 July 2011

Published 19 August 2011

Online at stacks.iop.org/JPhysCM/23/365501

\begin{abstract}
Calculations of the band structure, partial densities of states and optical spectra of permittivity, reflectivity and absorption of perfect $\mathrm{ZnMoO}_{4}$ crystal were performed using the full-potential linear-augmented-plane-wave method. It is shown that the calculated reflectivity spectra reproduce the main features of corresponding experimental spectra in the fundamental absorption region. The bandgap value of $\mathrm{ZnMoO}_{4}$ is estimated as $E_{\mathrm{g}}=4.3 \mathrm{eV}$. Peculiarities of luminescence excitation spectra corrected for near-surface losses and losses on reflectivity are discussed, taking into account the results of the calculations. It is found that the energy structure of the lower part of conduction band is manifested in the excitation spectra of the intrinsic luminescence. The excitation spectra in the region $4.3-8.0 \mathrm{eV}$ are formed by band-to-band electronic transitions mainly within the molybdate groups $\mathrm{MoO}_{4}^{2-}$, whereas electronic states of $\mathrm{Zn}^{2+}$ cations are not directly involved into the excitation processes. It is shown that the structure of the intrinsic luminescence excitation spectrum depends on the temperature and mechanisms of the structure modification are discussed.
\end{abstract}

(Some figures in this article are in colour only in the electronic version)

\section{Introduction}

Recently molybdate crystals have attracted attention as a novel material for cryogenic phonon-scintillation detectors [1]. Simultaneous detection of the scintillation light and phonon signal at very low temperatures (on the millikelvin scale) allows one to discriminate between $\alpha$ and $\beta$ particles and $\gamma$ radiation and to substantially reduce the background; all these together make this method suitable for searching for neutrinoless double beta decay and dark matter. Molybdate single crystals are considered as a material for cryogenic detectors due to the high light yield at low temperatures and also to the presence of molybdenum ${ }^{100} \mathrm{Mo}$, which is one of the most promising double beta decay sources [2]. The absence of heavy elements and a relatively high mass concentration of molybdenum in the crystal are apparent advantages of $\mathrm{ZnMoO}_{4}$ crystals that allow the low level of radioactive background required for cryogenic phonon-scintillating detector applications to be reached. The first encouraging results demonstrating the applicability of $\mathrm{ZnMoO}_{4}$ as a cryogenic detector were obtained recently [4].

Only a few papers deal with the investigation of $\mathrm{ZnMoO}_{4}$ luminescence. Luminescent properties have been studied for small single crystals grown by the method of spontaneous crystallization [3], and also for bulk crystals grown by the Czochralski method $[2,4,5]$ that were grown for the first time only recently. It was found that zinc molybdate belongs to the class of self-activated phosphors, i.e. it shows luminescence without additional doping of the crystal. Intrinsic emission of $\mathrm{ZnMoO}_{4}$ was ascribed to radiative annihilation of self-trapped excitons (STEs) on $\mathrm{MoO}_{4}^{2-}$ complexes, like in several other tungstates and molybdates of the $\mathrm{AXO}_{4}$ family $(\mathrm{A}=\mathrm{Ca}$, 
$\mathrm{Zn}, \mathrm{Cd}, \mathrm{Pb} ; \mathrm{X}=\mathrm{Mo}, \mathrm{W})$. Efficient energy transfer of the absorbed high-energy radiation to the emission centers is one of the most important factors to obtain a high light yield in a scintillator. Apparently it depends on the electronic band structure of the compound and the data on the electronic band structure allow for deeper insight into the energy transfer processes in $\mathrm{ZnMoO}_{4}$.

The electronic structures of several tungstates and molybdates of the $\mathrm{AXO}_{4}$ family were studied recently by several theoretical methods. It was shown that oxide crystals of this family have many congenial features in their electronic structures that are preconditioned by the presence of similar molecular oxyanions $\mathrm{XO}_{4}^{2-}$ in anionic positions of their lattice. The full-potential linear-augmented-plane-wave (FLAPW) method has been applied to studies of the electronic structures of perfect $\mathrm{ZnWO}_{4}$ [6], $\mathrm{PbWO}_{4}, \mathrm{CaWO}_{4}, \mathrm{CaMoO}_{4}$ and $\mathrm{PbMoO}_{4}$ [7], $\mathrm{CdWO}_{4}$ and $\mathrm{CdMoO}_{4}$ [8], as well as of $\mathrm{AXO}_{4}$ with several point defects $[9,10]$. Also the electronic structures of $\mathrm{PbWO}_{4}, \mathrm{CdWO}_{4}$ and $\mathrm{ZnWO}_{4}$ with point defects and impurities have been studied in the quantum chemical cluster approach [11-14]. The discrete variational $X \alpha$ method has been applied to the investigation of the electronic structure of $\mathrm{CdMoO}_{4}$ [15] and $\mathrm{AWO}_{4}$ with $\mathrm{A}=$ $\mathrm{Pb}, \mathrm{Ca}, \mathrm{Ba}, \mathrm{Cd}, \mathrm{Zn}$ [16]. The electronic band structure of perfect $\mathrm{ZnWO}_{4}$ has also been studied in the periodic Hartree-Fock formalism [17]. In our opinion, the FLAPW method provides the best agreement between calculated results and experimental data on the reflectance of $\mathrm{AXO}_{4}$ crystals in the VUV excitation region (see e.g. [6, 8]). Therefore we apply the FLAPW method for electronic structure calculations of perfect $\mathrm{ZnMoO}_{4}$.

In the present work the absorption and luminescence excitation of $\mathrm{ZnMoO}_{4}$ crystals is analyzed on the basis of the results of band structure calculations. The role of the regular elements of the crystal lattice $\left(\mathrm{Zn}^{2+}\right.$ cations and $\mathrm{MoO}_{4}^{2-}$ anions) in the luminescence excitation processes is clarified. The mechanisms that control the temperature behavior of the energy transfer efficiency to emission centers are discussed.

\section{Calculation methods and experimental details}

Zinc molybdate belongs to the $\alpha-\mathrm{ZnMoO}_{4}$ structural type, space group $P \overline{1}$, triclinic system $[18,19]$. The crystal structure of $\mathrm{ZnMoO}_{4}$ is presented in figure 1. The crystal is biaxial with the lattice constants $a=9.625 \AA, b=6.965 \AA, c=8.373 \AA$, $\alpha=103^{\circ} 28^{\prime}, \beta=96^{\circ} 30^{\prime}, \gamma=106^{\circ} 72^{\prime}$. The primitive unit cell contains six $\mathrm{ZnMoO}_{4}$ units. The molybdenum atoms are surrounded by distorted tetrahedra of oxygen atoms forming isolated $\mathrm{MoO}_{4}$ complexes. In total there are three different types of distorted $\mathrm{MoO}_{4}$ complexes in $\mathrm{ZnMoO}_{4}$. Zinc atoms are coordinated into the octahedral ( $\mathrm{Zn} 1$ and $\mathrm{Zn} 2$ in figure 1) and pentahedral $(\mathrm{Zn} 3)$ oxygen surroundings.

The electronic structure of the perfect zinc molybdate crystal $\mathrm{ZnMoO}_{4}$ was calculated using the WIEN2k package [20], in which the full-potential linear-augmented-planewave (FLAPW) method is implemented within the framework of density-functional theory (DFT). The Perdew and Wang generalized gradient approximation was employed [21] for the

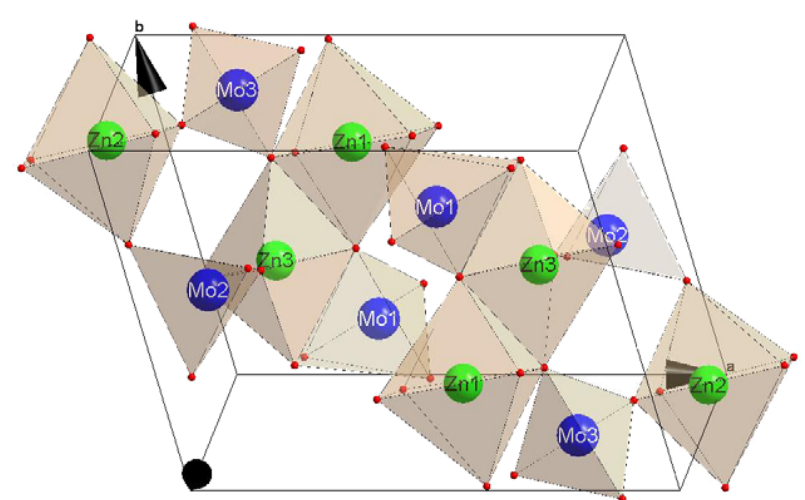

Figure 1. The unit cell of $\mathrm{ZnMoO}_{4}$. The data on the atom positions were taken from [18].

exchange-correlation potential. The relativistic effects were treated in the scalar relativistic approximation. The energy of separation between core and valence states was chosen as -6.0 Ryd. The muffin-tin (MT) radii $R_{\mathrm{MT}}$ were chosen from the condition of 'almost touching' MT spheres as 1.94, 1.71 and 1.52 au for $\mathrm{Zn}$, Mo and $\mathrm{O}$ respectively. The potential and charge density in the MT spheres were expanded in spherical harmonics with $l_{\max }=10$. The plane wave cutoff parameter $R_{\mathrm{MT}} K_{\max }$ was equal to 6.0. The magnitude of the largest vector $G_{\max }$ in the charge density Fourier expansion was 14.0.

The convergence criterion was chosen as the 0.0001 Ryd convergence of total energy. The modified tetrahedron method was used for the Brillouin zone (BZ) integration [22]. The densities of electronic states (DOS) and optical constants were calculated using well-known relations implemented in the WIEN2k program code [20]. The calculations were carried out for 40, 50 and 60 nonequivalent sampling $\boldsymbol{k}$-points, and it was found that further increase of the number of $\boldsymbol{k}$-points does not practically change the results of the calculations. In this paper we present the results obtained for $60 k$-points in the irreducible part of the BZ.

Absorption and reflectance spectra in the fundamental absorption region of nominally pure crystals should not depend on the particular sample, since they are determined by fundamental optical constants. However, the reflectivity and luminescence excitation spectra obtained in the experiment are usually sample-dependent. For the reflectivity the anisotropy of the crystal structure should be taken into account. Actually the profile of reflectivity may substantially depend on the orientation of the sample during the measurements. The luminescence excitation spectra depend on imperfections of various origins (defects, inadvertent impurities, etc) introduced into real crystals during crystal growth. In order to exclude the influence of such irregularities of the crystal structure to the greatest possible extent, we performed the measurements on two nominally pure $\mathrm{ZnMoO}_{4}$ crystalline samples grown by different methods, namely the Czochralski (sample \#1) and Kiropulos (sample \#2) techniques. The details of the crystal growth were described in [23]. Both samples have yellowish coloration. The $\mathrm{x}$-ray phase analysis confirmed the triclinic phase of both investigated samples. 


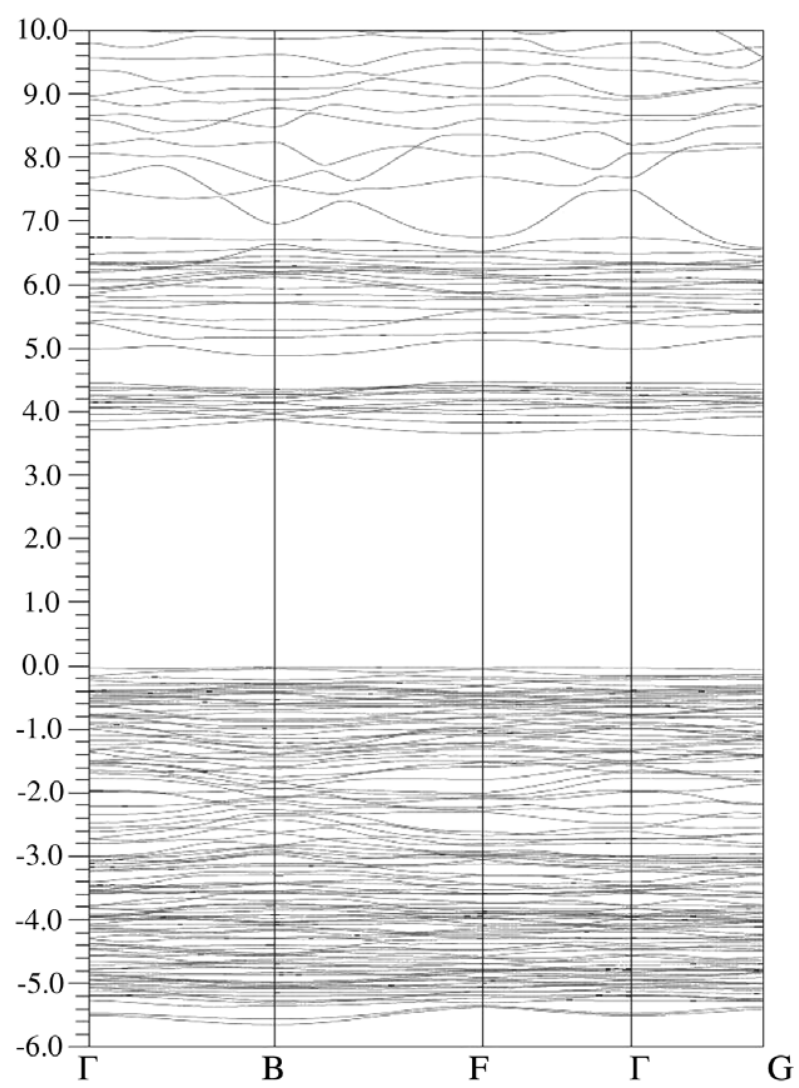

Figure 2. The calculated band structure of $\mathrm{ZnMoO}_{4}$.

Luminescence excitation and reflectivity spectra were measured at the SUPERLUMI station (DESY, Hamburg) [24] in the temperature range $10-300 \mathrm{~K}$. The measurements were carried out on freshly cleaved plane surfaces of samples corresponding to the crystallographic plane $\left.\begin{array}{llll}0 & 0 & 1\end{array}\right\}$. Sample \#1 was oriented along the crystallographic axes using the electron backscatter diffraction method on an Oxford Instruments INCA 'CRYSTAL' system. The projection of the electric vector $\mathbf{E}$ of the incident synchrotron radiation on the sample surface was parallel to the crystallographic plane $\left\{\begin{array}{lll}2 & 3 & 0\end{array}\right\}$ for sample \#1. The reflectivity of sample \#2 was measured at a random orientation of $x$ and $y$ axes.

\section{Electronic band structure, densities of states and reflectance spectra of $\mathrm{ZnMoO}_{4}$}

The energy dispersion curves $E_{k}$ (one-electron bands) calculated for four directions that connect the special $\boldsymbol{k}$-points $\Gamma, \mathrm{B}, \mathrm{F}$ and $\mathrm{G}$ of the Brillouin zone of the triclinic lattice are presented in figure 2. One-electron bands are in general uniformly distributed along the valence band (VB), excluding the region between -3 and $-1.5 \mathrm{eV}$, where they are packed more sparsely. As follows from calculations, the conduction band $(\mathrm{CB})$ minimum $(3.62 \mathrm{eV})$ is reached at the $\mathrm{G}$ point, the $\mathrm{VB}$ maximum $(0 \mathrm{eV})$ can be found in the $\mathrm{F}-\Gamma$ section. The calculated $E_{\mathrm{g}}$ value equals $3.76 \mathrm{eV}$ at the $\Gamma$ point. So, $\mathrm{ZnMoO}_{4}$ crystal can be considered as an indirect-gap material with $0.14 \mathrm{eV}$ difference between the indirect band gap and

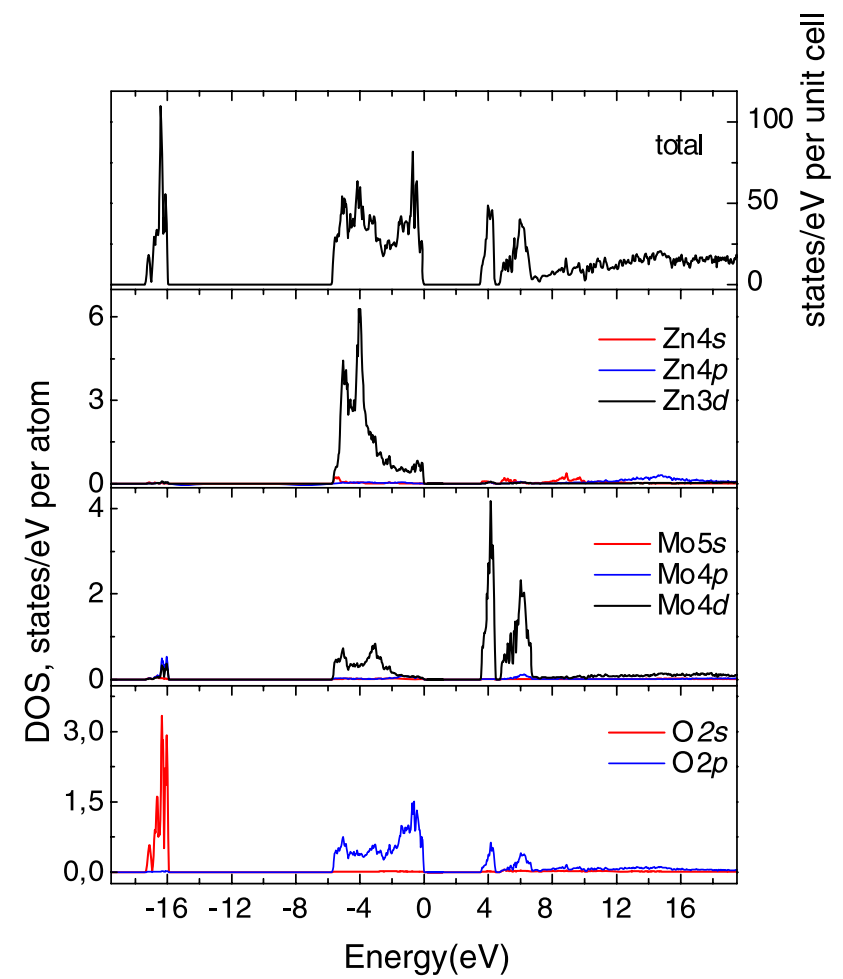

Figure 3. The calculated total and partial density of states of $\mathrm{ZnMoO}_{4}$

$E_{\mathrm{g}}$ at the $\Gamma$ point. The dispersion of one-electron bands of $\mathrm{ZnMoO}_{4}$ is in general close to the case of $\mathrm{CaMoO}_{4}$, which is a direct-gap material [9], and, at the same time, it is significantly lower than for $\mathrm{PbMoO}_{4}$, where the difference between direct and indirect gaps reaches $\sim 1 \mathrm{eV}$ [7].

The calculated total and partial densities of states (PDOS) of $\mathrm{ZnMoO}_{4}$ are presented in figure 3. The origin of the energy

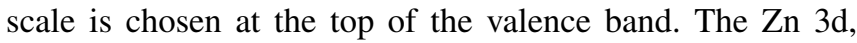
Mo $4 \mathrm{~d}$ and $\mathrm{O} 2 \mathrm{p}$ form the VB of the crystal which ranges from -5.8 to $0 \mathrm{eV}$. The contribution of the other types of electronic states to the VB is insignificant. The $\mathrm{O} 2$ s core band is located $10 \mathrm{eV}$ below the bottom of the $\mathrm{VB}$, from -17.4 to $-15.9 \mathrm{eV}$. The Mo 4p states also have some contribution to this core band.

The existence of two regions of relatively high density of states (3.5-4.4 and 4.7-6.7 eV, see figure 3) is a distinctive feature of the $\mathrm{CB}$ of $\mathrm{ZnMoO}_{4}$. As figure 2 shows, these two regions contain dense groups of one-electron bands with a relatively low dispersion, which are separated by a $0.3 \mathrm{eV}$ gap with zero density. The first region is composed of 12 one-electron bands, whereas the second one has 18 such bands. One-electron bands are distributed more sparsely in the upper part of the CB. The Mo $4 \mathrm{~d}$ states (with some contribution from $\mathrm{O} 2 \mathrm{p}$ states) clearly dominate in the $\mathrm{CB}$ below $6.7 \mathrm{eV}$, and the splitting between these subbands corresponds to $E-T$ splitting of Mo 4 d states in the tetrahedral crystal field (with account for six $\mathrm{MoO}_{4}^{2-}$ groups per unit cell). The $\mathrm{Zn} 3 \mathrm{~d}$ states are practically absent in the $\mathrm{CB}$; however, some contribution of $\mathrm{Zn} 4 \mathrm{~s}$ and $\mathrm{Zn} 4 \mathrm{p}$ can be distinguished in this energy region below and above $10 \mathrm{eV}$ respectively. 


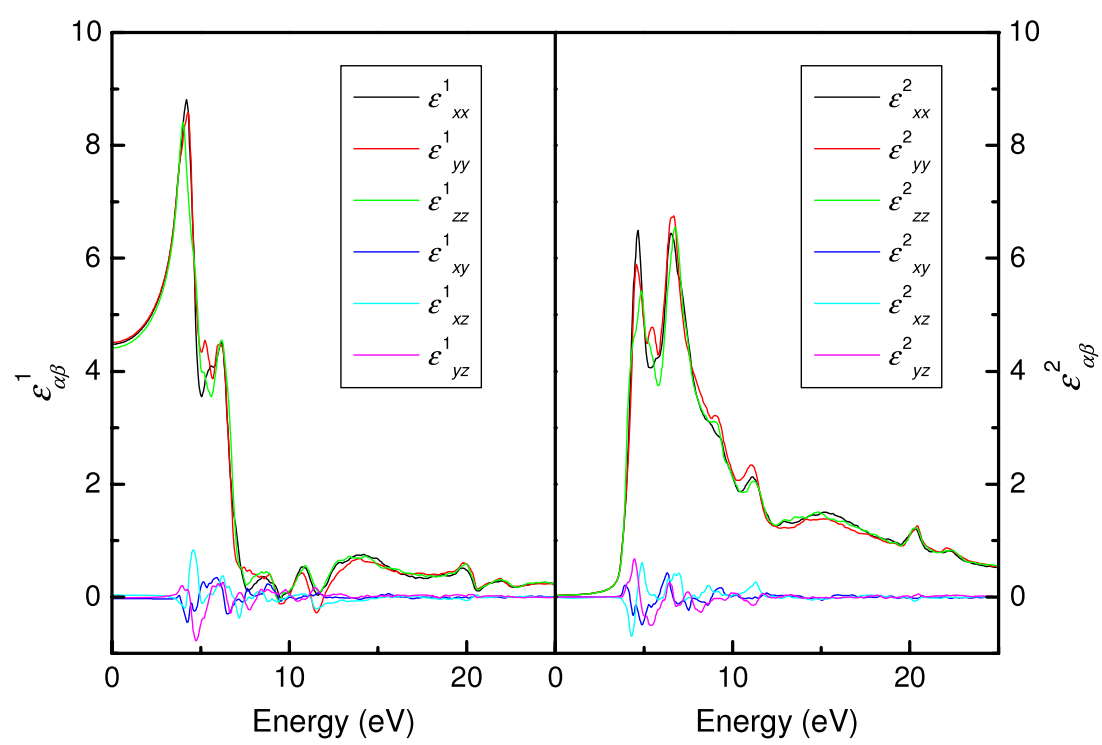

Figure 4. The calculated real and imaginary parts of the dielectric tensor of $\mathrm{ZnMoO}_{4}$.

The top of the VB is formed by $\mathrm{O} 2 \mathrm{p}$ and $\mathrm{Zn} 3 \mathrm{~d}$ states without significant contribution from Mo $4 \mathrm{~d}$ states. As was shown recently by electronic structure calculations, such absence of $\mathrm{W}$ (Mo) states at the top of the VB is characteristic of tungstate and molybdate crystals, e.g. of $\mathrm{CdMoO}_{4}$ [8], $\mathrm{CaMoO}_{4}, \mathrm{PbWO}_{4}$ [9], $\mathrm{CaWO}_{4}$ and $\mathrm{PbMoO}_{4}$ [7]. This feature is typical for the electronic structure of transition metal complexes $\mathrm{XO}_{4}^{2-}(\mathrm{X}=\mathrm{Cr}, \mathrm{Mo}, \mathrm{W})$. Almost all methods of electronic structure calculations show that the highest occupied molecular orbital of such a complex is formed purely by $\mathrm{O} 2 \mathrm{p}$ states $[9,25,26]$. The contribution of $\mathrm{Zn} 3 \mathrm{~d}$ states increases in the lower part of the VB. Two notable peaks arise near -4 and $-5 \mathrm{eV}$ in the $\mathrm{Zn} 3 \mathrm{~d}$ DOS distribution.

It should be noted that the $E_{\mathrm{g}}$ value obtained directly from our calculations should be somewhat corrected using experimental data. It is known that the band gap of a crystal may be underestimated if calculations are made under the DFT formalism [27]. To overcome this drawback, the correcting energy addition to $E_{\mathrm{g}}$ or the 'scissor operator' $\Delta$ is introduced at the stage of calculation of the optical parameters. Then the value of $\Delta$ is obtained from the best fit between the calculated optical spectra and the corresponding experimental data. After this fitting, such a corrected $E_{\mathrm{g}}$ value is accepted as a real band gap of a crystal. Here we apply this procedure to the case of $\mathrm{ZnMoO}_{4}$ crystal.

The components of the dielectric tensor were calculated using the relations [28]

$$
\begin{gathered}
\varepsilon_{\alpha \beta}^{2}(\hbar \omega)=\frac{4 \pi e^{2}}{m^{2}(\hbar \omega+\Delta)^{2}} \sum_{c, v} \int \mathrm{d} \vec{k}\left\langle\Psi_{c \vec{k}}\left|p_{\alpha}\right| \Psi_{v \vec{k}}\right\rangle \\
\times\left\langle\Psi_{v \vec{k}}\left|p_{\beta}\right| \Psi_{c \vec{k}}\right\rangle \delta\left(E_{c \vec{k}}-E_{v \vec{k}}-\hbar \omega+\Delta\right), \\
\varepsilon_{\alpha \beta}^{1}(\hbar \omega)=\delta_{\alpha \beta}+\frac{2}{\pi} P \int_{0}^{\infty} \frac{\omega^{\prime} \varepsilon_{\alpha \beta}^{2}\left(\hbar \omega^{\prime}\right)}{\omega^{\prime 2}-\omega^{2}} \mathrm{~d} \omega^{\prime},
\end{gathered}
$$

where $\Psi_{\vec{v}}, \Psi_{c \vec{k}}$ are calculated one-electron wavefunctions; $E_{v \vec{k}}, E_{c \vec{k}}$ are calculated eigenvalues, corresponding to the VB and $\mathrm{CB}$ respectively, $\Delta$ is the scissor operator; $\alpha, \beta=x, y, z$.

When the principal dielectric axes of a crystal coincide with the axes $x, y, z$ of the chosen reference frame, the Fresnel equations are significantly simplified and the reflectance and absorbance spectra can be calculated using relations that utilize only diagonal components of the dielectric tensor $\varepsilon_{\alpha \alpha}$. In the crystals of a triclinic system, to which $\mathrm{ZnMoO}_{4}$ belongs, the directions of the principal dielectric axes are not related to any crystallographic axes. In this case the relations for reflectivity and absorption coefficients become very complicated [29]. Figure 4 presents results for dielectric tensor components of $\mathrm{ZnMoO}_{4}$ calculated using relations (1) and (2) for the case of $\Delta=0$. It is clearly seen that the off-diagonal components $\varepsilon_{\alpha \beta}, \alpha \neq \beta$ of the tensor are an order of magnitude less than the diagonal ones. This fact indicates that the directions of the dielectric principal axes of the crystal do not differ substantially from the $x, y, z$ axes of the chosen coordinate system. This gives us a reason to disregard the off-diagonal components in the calculations of reflectance and absorption coefficients. The corresponding relations in the case of normal incidence of a light beam are the following:

$$
\begin{gathered}
R_{\alpha \alpha}(\hbar \omega)=\frac{\left(n_{\alpha \alpha}-1\right)^{2}+\kappa_{\alpha \alpha}^{2}}{\left(n_{\alpha \alpha}+1\right)^{2}+\kappa_{\alpha \alpha}^{2}} \quad \text { and } \\
K_{\alpha \alpha}(\hbar \omega)=\frac{2 \omega}{c} \kappa_{\alpha \alpha}
\end{gathered}
$$

where

$$
\begin{aligned}
& n_{\alpha \alpha}(\hbar \omega)=\sqrt{\frac{\left|\varepsilon_{\alpha \alpha}(\hbar \omega)\right|+\varepsilon_{\alpha \alpha}^{1}(\hbar \omega)}{2}}, \\
& \kappa_{\alpha \alpha}(\hbar \omega)=\sqrt{\frac{\left|\varepsilon_{\alpha \alpha}(\hbar \omega)\right|-\varepsilon_{\alpha \alpha}^{1}(\hbar \omega)}{2}} .
\end{aligned}
$$

Reflectivity spectra for the $x$-, $y$-, and $z$-polarizations of the $\mathbf{E}$ vector of incident light calculated with $\Delta=0.7 \mathrm{eV}$ 


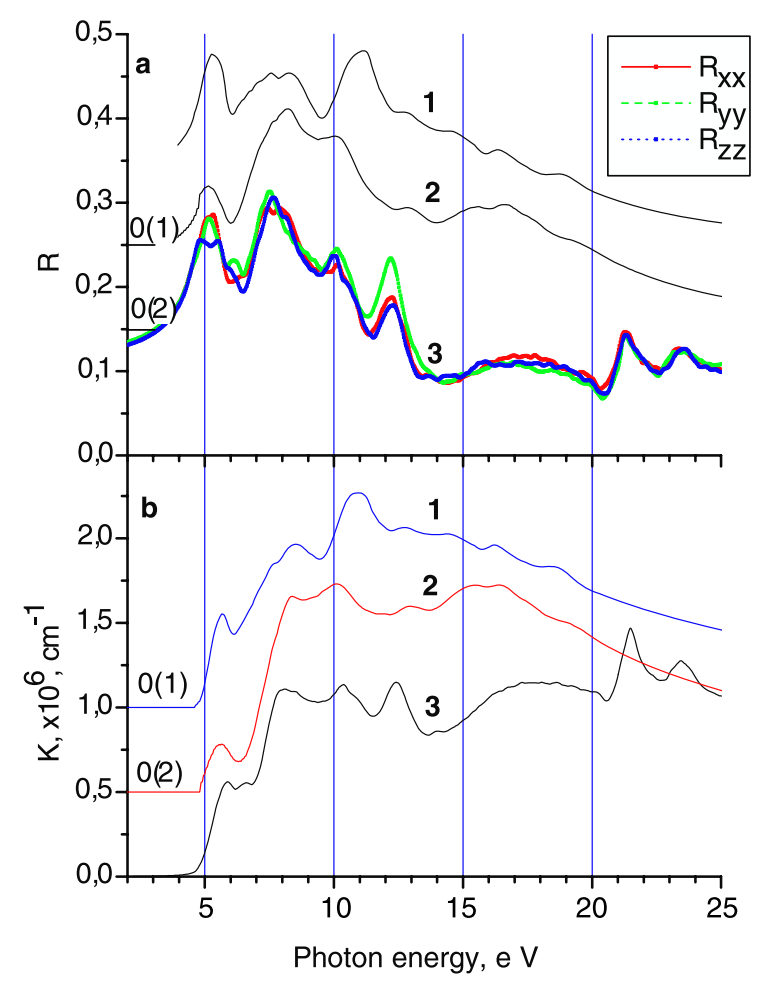

Figure 5. Top plot: experimental reflectivity spectra $(T=10 \mathrm{~K})$ of $\mathrm{ZnMoO}_{4}$ sample \#1 (curve 1), sample \#2 (curve 2) and calculated (group of curves denoted by 3 ) reflectivity spectra for the $x$-, $y$-, and $z$-polarizations of the $\mathbf{E}$ vector of incident light. Bottom plot: absorption spectra calculated using Kramers-Kronig relations from the experimental reflectivity for samples \#1 and \#2 (curves 1 and 2) and the calculated absorption averaged in three directions: $K=\left(K_{x x}+K_{y y}+K_{z z}\right) / 3$ (curve 3 ).

are presented in figure 5(a) together with the experimental reflectivity measured for two samples of $\mathrm{ZnMoO}_{4}$ which were grown by two different methods. The value of $\Delta=0.7 \mathrm{eV}$ used for the calculations gives the best fit between calculated and experimental spectra.

As figure 5(a) shows, the main features of the experimental reflectivity are similar for both samples of $\mathrm{ZnMoO}_{4}$ : the first sharp peak at $5.2 \mathrm{eV}$; a dip at $6.0 \mathrm{eV}$; a broad non-elementary band in the $6.0-9.5 \mathrm{eV}$ region with a peak at $\sim 8.3 \mathrm{eV}$; a relatively weak peak at $12.9 \mathrm{eV}$; and a broad band in the $14-20 \mathrm{eV}$ region. The difference between the $\mathrm{ZnMoO}_{4}$ samples is manifested in the relative intensity of the first peak and position of the peak in the region 9.5-12.3 eV (11.1 eV for sample \#1 and $10.2 \mathrm{eV}$ for sample \#2). Accordingly the main features of the experimental reflectivity are well represented in the calculated spectra for all three polarizations in the region up to $10.5 \mathrm{eV}$.

It should be noted that the experimental and calculated reflectance spectra have some discrepancies. The peaks at $11.1 \mathrm{eV}$ for sample \#1 and at $12.9 \mathrm{eV}$ for both samples are not reproduced in the calculated spectra. The calculated spectra predict a peak at $12.2 \mathrm{eV}$, while the experimental ones have a dip at this energy. Neglecting the off-diagonal components $\varepsilon_{\alpha \beta}, \alpha \neq \beta$ can be one of the reasons for the observed discrepancy in the experimental and calculated reflectance spectra. Besides this, the structures (peaks and dips) of the calculated spectra above $20 \mathrm{eV}$ were not detected in the experiment. However, a weakly expressed shoulder in the $21-26 \mathrm{eV}$ region is observed in the experimental reflectance for sample \#1.

The choice of $\Delta$ value was verified in an additional way, by comparison of the absorption coefficients obtained from the theoretical and experimental data. For convenience of further analysis we use the absorption coefficient averaged over three directions: $K=\left(K_{x x}+K_{y y}+K_{z z}\right) / 3$. The averaged $K$ spectrum calculated by (3) and (4) is presented in figure 5(b) together with the absorption spectra of two samples of $\mathrm{ZnMoO}_{4}$. The latter were obtained from the experimental reflectivity using Kramers-Kronig relations (details of the approach used in this calculation can be found, e.g., in [30]).

As figure 5(b) shows, the calculated $K$ curve represents all the features of both experimental curves in the 4-8 eV region; however, it somewhat differs from them in peak intensities. Such inessential disagreement most probably originates from the discrepancies between the calculated and experimental reflectances in this region noted above. Besides this, the calculated $K$ spectrum practically coincides with the experimental one for sample \#2 in the $4-8 \mathrm{eV}$ region. Such similarity of the $K$ spectra supports the choice of the scissor operator value $\Delta=0.7 \mathrm{eV}$.

Above the region where calculated and experimental $K$ spectra have disagreement in peak intensities (12-14 eV), all three curves reveal analogous wide humps between 14 and $20 \mathrm{eV}$. Intense dipole-allowed transitions from the $\mathrm{O} 2 \mathrm{~s}$ core band to the $\mathrm{CB}$ of $\mathrm{ZnMoO}_{4}$ are manifested as sharp peaks in the calculated $K$ spectrum above $20 \mathrm{eV}$. These peaks are not directly seen in the experimental curves.

With the value of $\Delta=0.7 \mathrm{eV}$ the bandgap value of $\mathrm{ZnMoO}_{4}$ is determined as $E_{\mathrm{g}}=4.3 \mathrm{eV}$. It correlates well with the estimation of $E_{\mathrm{g}}$ presented in [5]. Actually the $E_{\mathrm{g}}$ value was estimated on the basis of the experimental spectra of luminescence excitation and reflectivity as $4.17 \mathrm{eV}<E_{\mathrm{g}}<$ $5.35 \mathrm{eV}$.

In the following section we will analyze the formation of the intrinsic luminescence excitation spectra of $\mathrm{ZnMoO}_{4}$ using results of the electronic structure calculations presented above.

\section{Analysis of the excitation spectra of the intrinsic luminescence of $\mathrm{ZnMoO}_{4}$}

It was found that the intrinsic emission of $\mathrm{ZnMoO}_{4}$ is represented by a broad band peaking at around $2.1 \mathrm{eV}$ at $10 \mathrm{~K}$ [5]. An additional weak luminescence band was also observed at $2.45 \mathrm{eV}$ in [3]. The composite structure of the luminescence was attributed either to the Jahn-Teller interaction effect or to the emission from nonequivalent $\mathrm{MoO}_{4}^{2-}$ groups. The intrinsic emission band(s) of $\mathrm{ZnMoO}_{4}$ is due to the radiative annihilation of STEs localized on $\mathrm{MoO}_{4}^{2-}$, similarly to other self-activated tungstates and molybdates of the $\mathrm{AXO}_{4}$ family $(\mathrm{A}=\mathrm{Ca}, \mathrm{Zn}, \mathrm{Cd}, \mathrm{Pb} ; \mathrm{X}=$ Mo, W) [7, 31, 32]. The luminescence emission originates from Franck-Condon transitions from localized excited states to the ground states of self-trapped Frenkel-type excitons 
localized on $\mathrm{MoO}_{4}^{2-}$ molecular anions. It is clear that the data on the band structure should be taken into account for careful analysis of the STE luminescence excitation spectra of $\mathrm{ZnMoO}_{4}$ in the fundamental absorption region.

Less successful attempts at direct comparison between the excitation spectra of intrinsic emission of $\mathrm{PbWO}_{4}$ and $\mathrm{CaWO}_{4}$ crystals with calculated total densities of states in their valence bands were made before [7]. Attempts were also made to simulate the excitation spectra of several oxides (including tungstates) on the basis of valence bands given by parabolic or step-wise functions [33]. The calculated band structure, in a certain approximation, provides information on the variety of possible initial and final states of the excited electron only at the moment 'just after' absorption of a photon with a given energy. Therefore experimental absorption or reflection spectra can be reproduced in calculations carried out in the band formalism since these spectra are determined directly by these initial and final states and do not depend on the further fate of the excited charge carriers. However, the formation of luminescence excitation spectra depends on the processes of transfer of electronic excitations. As a consequence, the relation between the STE luminescence excitation spectra of $\mathrm{ZnMoO}_{4}$ and the electronic structure of a crystal is a non-trivial problem that requires careful analysis. Not only the band structure itself, but also the processes of excitation energy transfer to the emission centers should be taken into account.

Excitation spectra of intrinsic emission are presented in figure 6(a) for $\mathrm{ZnMoO}_{4}$ sample \#1. Since the excitation spectra of both investigated samples were similar at all temperatures studied, further analysis will refer to sample \#1. The luminescence yield significantly depends on the excitation energy as well as on the temperature of the sample. According to our estimation of the band gap value (see section 3), the low-energy edge of the luminescence excitation $(3.9 \mathrm{eV}<E<4.3 \mathrm{eV})$ is located below the fundamental absorption region. When the excitation energy exceeds the $E_{\mathrm{g}}$ value $(4.3 \mathrm{eV})$, the band-to-band electronic transitions start to participate in the luminescence excitation process. The processes that may contribute to the formation of the luminescence excitation spectra in the fundamental absorption region and have to be accounted for together with the contributions from the features of the band structure are as follows.

(1) Losses on reflection and near-surface losses. In the energy region of the fundamental absorption the absorption coefficient reaches values of up to $10^{5}-10^{6} \mathrm{~cm}^{-1}$. Hence the elementary processes of photon absorption take place only in a narrow near-surface layer. If electronic excitations (electrons/holes or excitons) can migrate through the crystal, and the probability of their non-radiative annihilation at the surface (near-surface losses) is high enough, the features of the excitation spectrum can be anti-correlated with respect to the absorption (peaks in the excitation coincide with dips in the absorption and vice versa) [30]. The losses on reflection are obviously proportional to the value of

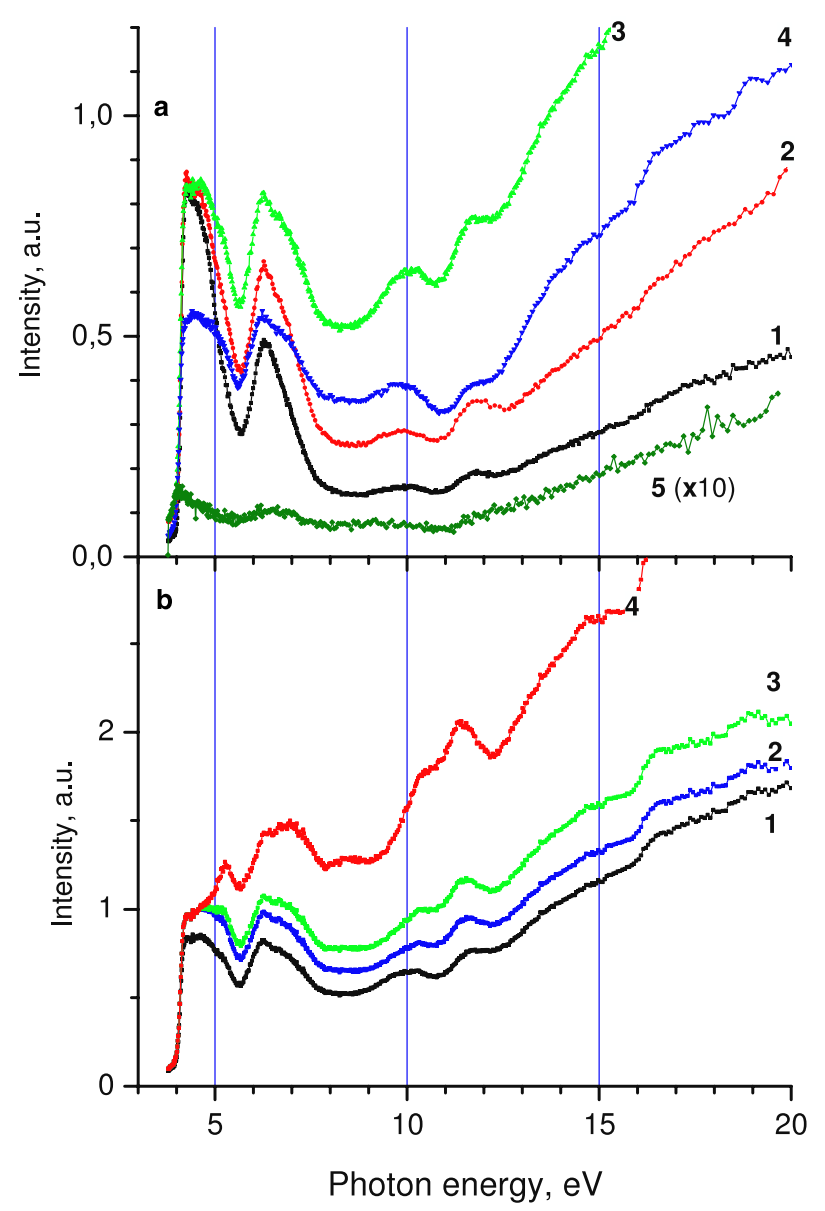

Figure 6. Top plot: excitation spectra of intrinsic luminescence $\left(E_{\mathrm{em}}=2.1 \mathrm{eV}\right)$ of $\mathrm{ZnMoO}_{4}$ sample $\# 1$ at $T=10,70,110,150$ and $300 \mathrm{~K}$ (curves $1-5$ respectively). Bottom plot: excitation spectra of intrinsic luminescence at $T=110 \mathrm{~K}$ without correction (curve 1), corrected for the reflectivity losses (curve 2 ), corrected for the reflectivity and near-surface losses for $L=2 \mathrm{~nm}$ (curve 3 ) and for $L=10 \mathrm{~nm}$ (curve 4 ).

reflectance, since photons reflected from the crystal do not participate in the luminescence excitation. These effects generally result in the 'modulation' of the excitation spectrum with the reflectivity and absorption spectra.

(2) At the excitation energies $E>2 E_{\mathrm{g}}$ the multiplication of the electronic excitations starts. At the multiplication stage one excitation photon may produce two (or more at higher energies) photons of the luminescence. Hence the process is usually observed as a rise of the luminescence intensity in the excitation spectrum.

(3) Presence of the competitive relaxation channels that affect the luminescence efficiency at the thermalization stage of energy relaxation. It will be shown below that the competitive relaxation channel leads to modification of the excitation spectrum in $\mathrm{ZnMoO}_{4}$ at low temperatures.

The near-surface losses and losses on reflection are taken into account by a simple diffusion theory developed for the case of diffusion-like migration of free excitons (see e.g. [30]). The measured luminescence excitation yield $I_{\text {exp }}$ in 
the fundamental absorption region can be given by the formula

$$
I_{\exp }(h v)=\frac{(1-R(h v))}{1+K(h v) L} \eta_{\mathrm{vol}},
$$

where $h v$ is the excitation photon energy, $R$ is the reflectivity, $K$ is the absorption coefficient, and $L$ is the diffusion length parameter (its value in our case will be discussed below). $L$ is the diffusion length over the lifetime $\tau$ of the electronic excitation responsible for the migration: $L=(D \tau)^{1 / 2}, D$ is the diffusion coefficient. The parameter $\eta_{\mathrm{vol}}$ is the luminescence quantum yield, usually called the 'volume quantum yield' since it is determined by the processes that actually take place in the crystal's bulk.

The exciton diffusion length $L$ is a parameter characteristic for the compound. The model of simple diffusion theory has been applied recently to determine the diffusion length of several dielectric crystals using relation (5) and experimentally measured reflectance spectra. It was found that its order of magnitude ranges from a few to hundreds of nanometers [34, 35]. Precise determination of $L$ is a non-trivial problem and requires additional studies [30]; however, we can assume that the value of $L$ for zinc molybdate should be comparatively low. As follows from the calculated band structure (figure 2), dispersion of electronic states in the vicinity of the bandgap is low. Low dispersion leads to massive electrons and holes that may predetermine low mobility of excitons in $\mathrm{ZnMoO}_{4}$. Besides this, the value of $L$ can be low due to the relatively short lifetime $\tau$ of free excitons before self-trapping. It is known that such a situation is realized in crystals with strong phonon coupling and the oxide crystals $\mathrm{AXO}_{4}$ are commonly attributed to this class of wide-gap insulators [7].

Experimental luminescence excitation spectra $I_{\text {exp }}$ measured at $110 \mathrm{~K}$ are presented in figure $6(\mathrm{~b})$ together with the $\eta_{\text {vol }}$ spectra calculated using relation (5) for different values of the diffusion length $L$. The experimental reflectivity $R$ was used for the calculation of $\eta_{\mathrm{vol}}$, while the absorption coefficient $K$ was obtained from the experimental $R$ using Kramers-Kronig relations (both $R$ and $K$ spectra are presented in figure 5).

When we account only for the energy losses on the reflectivity (no diffusion, $L$ is set to zero) the features of $\eta_{\text {vol }}$ structure that are anti-correlated to those in the reflectivity spectrum become less pronounced (figure 6(b), curve 2). If we also account for the near-surface losses ( $L$ is non-zero), further smoothing of the spectrum is observed. The optimal smoothing of the $\eta_{\mathrm{vol}}$ spectrum was obtained for the value of diffusion length $L=2 \mathrm{~nm}$ (curve 3). At higher values of $L$ (e.g. $L=10 \mathrm{~nm}$, figure 6(b), curve 4), new features appear in $\eta_{\mathrm{vol}}$ and the spectrum becomes distorted, while reasonable values of $L$ should not lead to significant distortions. Thus in the further discussion, we will use $\eta_{\mathrm{vol}}$ spectra calculated for $L=2 \mathrm{~nm}$.

The $\eta_{\mathrm{vol}}$ spectra at different temperatures are presented in figure 7(a) together with the partial densities of states. The band gap value in the DOS plot was corrected to $E_{\mathrm{g}}=$ $4.3 \mathrm{eV}$ in section 3 to obtain the best agreement between experimental and calculated reflectivity. A sharp rise of the
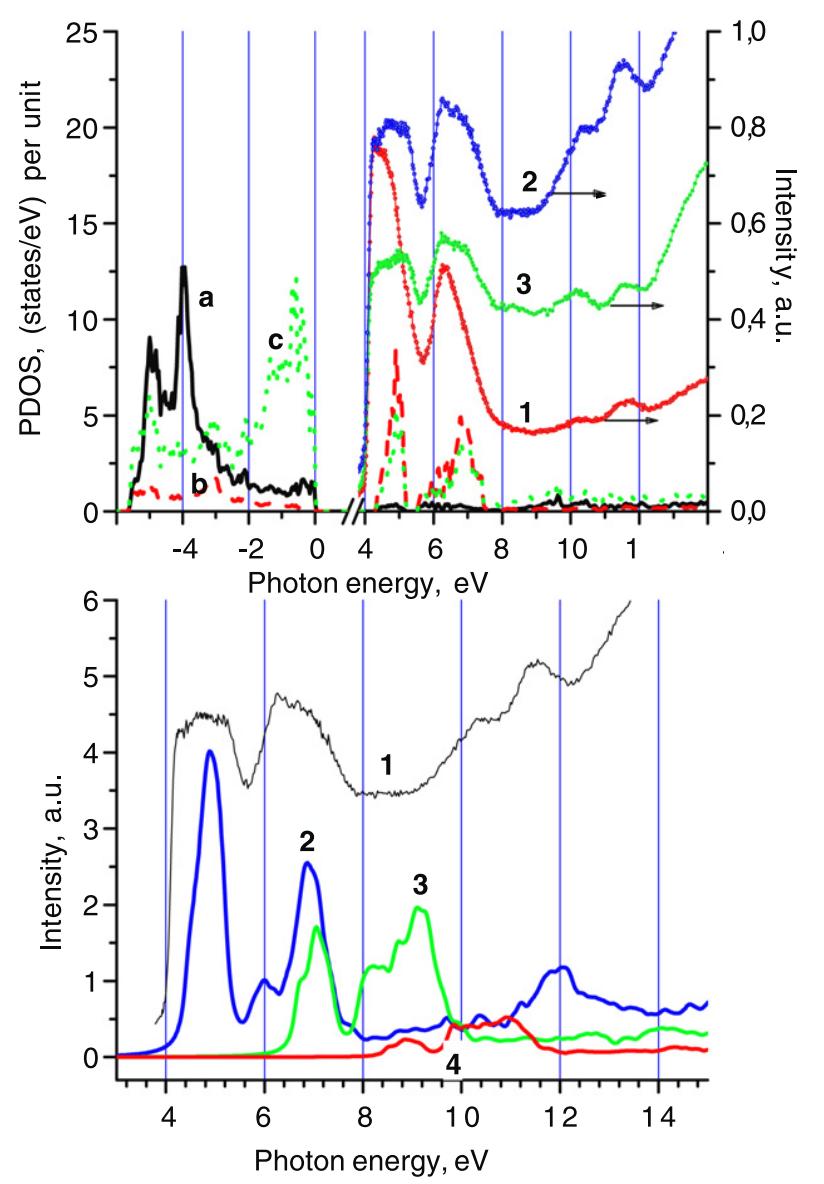

Figure 7. Top plot: partial densities of electronic states of $\mathrm{Zn}$ (curve a), Mo (curve b) and $\mathrm{O}$ (curve c) and $\eta_{\mathrm{vol}}$ of $\mathrm{ZnMoO}_{4}$ calculated for the diffusion length value $L=2 \mathrm{~nm}$ for $T=10$ (1), 110 (2) and $150 \mathrm{~K}$ (3). Bottom plot: $\eta_{\mathrm{vol}}$ for $T=110 \mathrm{~K}(1)$ and 'partial' absorption spectra $K_{\mathrm{TVB}}(2), K_{-2}$ (3) and $K_{-4}$ (4) calculated for transitions from single one-electron bands to the conduction band (see the text for details).

$\eta_{\mathrm{vol}}$ intensity is observed in the energy region 3.9-4.2 eV and the first peak of $\eta_{\mathrm{vol}}$ is located at $4.24 \mathrm{eV}$. The structure may be due to the excitation in the region of direct creation of excitons that is not considered in DOS calculations. It was shown previously that the steepness and temperature shift of the fundamental absorption edge obey the Urbach rule that implies the existence of excitons in $\mathrm{ZnMoO}_{4}$ [5]. The position of the exciton maximum in $\mathrm{ZnMoO}_{4}$ was calculated as $4.17-4.19 \mathrm{eV}$ and it is close to the position of the first maximum of $\eta_{\mathrm{vol}}$ at $4.24 \mathrm{eV}$. This peak is supposed to be the manifestation of the exciton in $\mathrm{ZnMoO}_{4}$. In this case, the exciton binding energy in $\mathrm{ZnMoO}_{4}$ can be estimated as $\sim 60 \mathrm{meV}$. The value of the binding energy is typical for excitons in compounds with ion-covalent bonds. Since the top of the VB is formed mainly by the $\mathrm{O} 2 \mathrm{p}$ states and the bottom of the $\mathrm{CB}$ by the Mo $4 \mathrm{~d}$ states the exciton should be created within the $\mathrm{MoO}_{4}^{2-}$ molecular anion. The extent of the low-energy tail of the exciton peak is determined by the exciton-phonon interaction and depends on the slope coefficient $\sigma$ [36]. The width of the tail in $\mathrm{ZnMoO}_{4}(0.34 \mathrm{eV})$ is typical for tungstates and molybdates where luminescence 
of self-trapped excitons is observed, i.e. $\mathrm{ZnWO}_{4}(0.4 \mathrm{eV})$, $\mathrm{MgWO}_{4}$ (0.35), $\mathrm{PbMoO}_{4}(0.2)$ [7, 37, 38]. It should be noted that there are no sharp peaks in the reflectivity of $\mathrm{ZnMoO}_{4}$ that can be considered as characteristic excitonic peaks (figure 5(a)). As was shown by the calculations of one-electron bands, $\mathrm{ZnMoO}_{4}$ has an indirect bandgap (figure 3). It is well known that excitons formed in an indirect electron transition are usually not manifested in the reflectivity spectra.

The energy position of the lower subband of CB conforms to the high-energy part of the first peak in $\eta_{\text {vol }}$. With further increase of the excitation energy $\eta_{\mathrm{vol}}$ reaches a minimum in the 5.5-5.7 eV region. The energy position of the minimum in $\eta_{\mathrm{vol}}$ correlates with the position of the gap between two subbands of the CB (5.6-5.9 eV). The position and width of the second broad band in $\eta_{\mathrm{vol}}(5.7-7.7 \mathrm{eV})$ also correlate with the energy position of the upper subband of the CB. A minimum in $\eta_{\mathrm{vol}}$ at $7.9 \mathrm{eV}$ conforms to a drastic drop of the density of states in the CB. In other words, the main features of the CB structure are directly manifested in $\eta_{\mathrm{vol}}$. This may imply that the electronic transitions from the top of the valence band (TVB) to the CB should dominate in excitation processes of $\mathrm{ZnMoO}_{4}$ intrinsic emission.

In order to confirm this assumption, we have calculated the 'partial' spectrum of the absorption coefficient $K_{\mathrm{TVB}}$ (figure 7(b), curve 2) in which only the electronic transitions from the highest occupied (HO) one-electron band (the top of the valence band) to all unoccupied one-electron bands (i.e. to the CB) are considered. The same relations ((1)-(4)) were used in calculations of $K_{\mathrm{TVB}}$ as for the 'total' $K$ spectra, in which transitions from all occupied one-electron bands are accounted for. Manifestation of $K_{\mathrm{TVB}}$ in the excitation spectra $\eta_{\text {vol }}$ (see figure 7(b)) means that, among all possible band-to-band transitions that can be realized at a given excitation energy, mainly transitions from the TVB create electrons and holes that afterward are bound into excitons that undergo subsequent radiative annihilation and cause intrinsic emission. If, in addition, we take into account that the oxygen states dominate in the TVB and the molybdenum states prevail in the $\mathrm{CB}$ (at least below $\sim 9 \mathrm{eV}$ ), it should be assumed that excitation of intrinsic emission takes place by means of generic electrons and holes that are bounded into excitons within the same $\mathrm{MoO}_{4}^{2-}$ groups. Electrons before binding into excitons should be thermalized mainly within the states of the molybdenum ions located in the centers of the deformed tetrahedra of oxygen ligands (see figure 1). A hole component of the exciton is initially created in the TVB and has mainly the oxygen character. However the probability for separated charge carriers to be bound into excitons in $\mathrm{ZnMoO}_{4}$ at $T=$ $110 \mathrm{~K}$ is also high at energies well above $E_{\mathrm{g}}$. This inference can be obtained from the fact that the intensity level of $\eta_{\mathrm{vol}}$ at energies where separated electrons and holes are created is generally the same as in the region of the direct exciton creation (near $E_{\mathrm{g}}$ ).

It should be noted that the comparatively low density of $\mathrm{Zn}$ states at the TVB and in the lower part of the CB is in accordance with the results of the electronic structure calculations carried out by different methods for $\mathrm{ZnWO}_{4}[6$,
17] and other $\mathrm{AXO}_{4}$ crystals with relatively 'light' A cations, e.g. $\mathrm{Ca}$ and $\mathrm{Cd}[7,8]$. On the basis of experimental data on photostimulated recombination luminescence, it was shown that electronic states of $\mathrm{XO}_{4}^{2-}$ molecular anions of such crystals actively participate in excitation of intrinsic emission in the region of excitation energies up to $\sim 1.5 \mathrm{eV}$ above the fundamental absorption edge [39, 40]. The A cations have considerably higher contribution at the TVB in $\mathrm{AXO}_{4}$ compounds with 'heavy' cations, namely $\mathrm{Pb}[7,11,16]$, and therefore an active participation of the $\mathrm{Pb}$ states in excitation of intrinsic emission is considered to be highly probable [38, 41, 42].

The role of band-to-band electronic transitions from deeper regions of the $\mathrm{VB}$ in the luminescence excitation processes in $\mathrm{ZnMoO}_{4}$ is considered below. The dips in $\eta_{\mathrm{vol}}$ at $\sim 5.5$ and $\sim 8 \mathrm{eV}$ are smeared out in comparison to the dips of the $K_{\mathrm{TVB}}$ spectrum. This means that transitions from the lower-lying electronic states of the VB also contribute to the $\eta_{\mathrm{vol}}$ formation. In order to analyze the possible contribution of transitions from 'deeper' states of the VB into the excitation spectra we have calculated some additional 'partial' absorption spectra using the same scheme as for $K_{\text {TVB }}$. One of them (denoted as $K_{-2}$ ) accounts only for electronic transitions from a single one-electron band located near $-2 \mathrm{eV}$ (the 39th band in order if counted from the $\mathrm{HO}$ band to lower energies, figure 3 ) to all one-electron bands of the CB. Another one (denoted as $\mathrm{K}_{-4}$ ) analogously accounts for all transitions to the $\mathrm{CB}$ from a single one-electron band located near $-4 \mathrm{eV}$ (the 71st band in order). As figure 7(a) shows, the $\mathrm{Zn}$ states dominate in the $\mathrm{VB}$ at $-4 \mathrm{eV}$ and therefore the $K_{-4}$ spectrum provides information on the spectral contribution of band-to-band transitions that have mainly the $\mathrm{Zn}$ character of initial states (i.e. the hole just after transition is located on the $\mathrm{Zn}$ states). The 'partial' absorption spectra $K_{-2}$ and $K_{-4}$ are presented in figure 7(b) together with the $K_{\mathrm{TVB}}$ spectrum and $\eta_{\mathrm{vol}}$ obtained at $T=110 \mathrm{~K}$. Obviously a correlation between $K_{-2}$ and $\eta_{\text {vol }}$ spectra is not observed, as the intensive band in the $K_{-2}$ spectrum between 8 and $10 \mathrm{eV}$ is not manifested in $\eta_{\mathrm{vol}}$. Thus the participation of electron transitions from the initial state at $-2 \mathrm{eV}$ below the TVB in the formation of excitation spectra is not as pronounced as that of the transitions of 'TVB-CB' type. Also $\eta_{\mathrm{vol}}$ does not reveal any intensive peaks in the $8-12 \mathrm{eV}$ region where $K_{-4}$ is non-zero. This might be indicative of the minor role played by the electronic states of the zinc cations in the process of excitation of intrinsic emission.

At $E_{\text {exc }}>12.2 \mathrm{eV}$ and beyond up to the high-energy limit of the measurements $(20 \mathrm{eV})$ the intensity of $\eta_{\mathrm{vol}}$ considerably increases. As the excitation energy exceeds the value of $2 E_{\mathrm{g}}$ the photon multiplication process is supposed to be responsible for the rise. According to band structure calculations the width of the valence band is larger than the bandgap value. This implies that the multiplication may occur not only through the high-energy electrons but also due the relaxation of the holes from the bottom of the valence band via the Auger process. The possibility of multiplication via hot holes was previously supposed to occur in oxides with wide valence bands, e.g. in borates [43]. The additional channel 
of creation of secondary low-energy electron-hole pairs due to the high-energy holes can be an advantageous feature of $\mathrm{ZnMoO}_{4}$ as a scintillator since it should increase the light yield in contrast to some other molybdates where $E_{\mathrm{g}} \geq E_{\mathrm{V}}$. For instance, in the well-known scintillating crystal $\mathrm{CaMoO}_{4}$ the width of the valence band is $\sim 5.0 \mathrm{eV}$ while the bandgap value is $\sim 5.1 \mathrm{eV}[7,44]$.

Analysis of the temperature dependence of $\eta_{\mathrm{vol}}$ is presented below. As figure 7(a) shows, $\eta_{\text {vol }}$ significantly depends on the temperature. In the whole energy range $\eta_{\mathrm{vol}}$ decreases at $T=150 \mathrm{~K}$ by a factor of $\sim 1.5$ in comparison with its values at $110 \mathrm{~K}$. With further heating to $T=300 \mathrm{~K} \eta_{\text {vol }}$ also decreases uniformly in the whole energy range by $\sim 50$ times in comparison with the yield at $T=110 \mathrm{~K}$. Thermal quenching in $\mathrm{ZnMoO}_{4}$ is known to start at $T>110 \mathrm{~K}$ [2-5] with the activation energy of the process $E_{\mathrm{act}}=117 \mathrm{meV}$. The thermal quenching is due to the increased probability of non-radiative relaxation of the already created STE. It is independent of the excitation energy leading to the decrease of $\eta_{\mathrm{vol}}$ at $T>110 \mathrm{~K}$.

At $T<110 \mathrm{~K}$ the profile of $\eta_{\mathrm{vol}}$ significantly depends on the temperature. $\eta_{\mathrm{vol}}$ is constant when the sample is cooled down from 110 to $10 \mathrm{~K}$ only in the region of direct exciton creation (first peak at $4.24 \mathrm{eV}$ ). For the excitation energies exceeding the bandgap, cooling below $110 \mathrm{~K}$ results in a decrease of $\eta_{\text {vol }}$ (see figure $7(\mathrm{a})$ ). The higher the excitation energy, the more pronounced this decrease is: in the region of the second broad band at $6.3 \mathrm{eV} \eta_{\mathrm{vol}}$ decreases only by a factor of 1.7 , while in the 8 to $12 \mathrm{eV}$ region it decreases by $\sim 4$ times with cooling of the sample from 110 to $10 \mathrm{~K}$.

We assume the following mechanism to be responsible for the decrease of $\eta_{\mathrm{vol}}$ with the decrease of temperature at energies above the band gap. Increase of the excitation energy leads to increase of the spatial separation of geminate electrons and holes after their thermalization. During migration separated electrons and holes can be captured by the competitive relaxation channels. Previously it was shown that the non-radiative relaxation of electronic excitations that competes with intrinsic emission exists in $\mathrm{ZnMoO}_{4}$ at low temperatures [5]. Traps which are responsible for the non-elementary peak of thermostimulated luminescence with the maximum at $55 \mathrm{~K}$ may act as a competitive channel of relaxation. When the temperature increases from 10 to $110 \mathrm{~K}$, thermal release of charge carriers from the traps occurs and the probability for separated electrons and holes to be bound into excitons (with subsequent self-trapping and radiative annihilation, which cause intrinsic emission) accordingly increases. Thus the relative contribution of the transitions with higher energies into the excitation spectrum is higher at $110 \mathrm{~K}$ than at $10 \mathrm{~K}$.

Finally it should be noted that the assumptions on the mechanisms of energy transfer to luminescence centers in $\mathrm{ZnMoO}_{4}$ are based on a somewhat original approach, namely on comparison of the calculated electronic structure with experimental excitation spectra. In future we plan to extend this approach to other substances.

\section{Conclusions}

Calculations of the band structure, partial densities of states and optical spectra of permittivity, reflectivity and absorption of perfect $\mathrm{ZnMoO}_{4}$ crystal were performed using the full-potential linear-augmented-plane-wave method. It is shown that the calculated reflectivity spectra reproduce the main features of the corresponding experimental spectra in the fundamental absorption region. The bandgap $E_{\mathrm{g}}$ of $\mathrm{ZnMoO}_{4}$ is determined as $4.3 \mathrm{eV}$.

Luminescence excitation spectra of $\mathrm{ZnMoO}_{4}$ corrected for reflection and near-surface losses $\left(\eta_{\mathrm{vol}}\right)$ were analyzed from the viewpoint of the aforesaid calculations. It is found that the most pronounced features of the density of electronic states in the conduction band of the crystal are manifested in $\eta_{\mathrm{vol}}$. The structure of $\eta_{\mathrm{vol}}$ in the energy region $4.3-8.0 \mathrm{eV}$ is determined by electronic transitions in $\mathrm{MoO}_{4}^{2-}$ oxyanionic groups, videlicet from the top of the valence band formed mainly of $\mathrm{O} 2 \mathrm{p}$ states to the conduction band where Mo $4 \mathrm{~d}$ states dominate. The role of the electronic states of $\mathrm{Zn}^{2+}$ cations in the formation of the $\eta_{\mathrm{vol}}$ spectra is found to be inessential.

It is found that thermal quenching of luminescence at $T>110 \mathrm{~K}$ and the competition of non-radiative relaxation with intrinsic emission at $T<110 \mathrm{~K}$ are the main factors that determine the temperature modifications of the excitation spectra of intrinsic emission in $\mathrm{ZnMoO}_{4}$.

\section{Acknowledgments}

Financial support by grants from the Federal Agency of Science and Innovations No 02.740.11.0546, BMBF Project RUS 10/037 and RFBR 11-02-01506-a is gratefully acknowledged. We are grateful to Professor G Zimmerer for providing the opportunity to perform measurements at the SUPERLUMI station and to Drs G Stryganyuk and A Kotlov for their assistance during the measurements. We are also grateful to Dr L Iskhakova for providing the x-ray phase analysis data and for orientation of the samples.

\section{References}

[1] Mikhailik V B and Kraus H 2006 J. Phys. D: Appl. Phys. 39 1181-91

Pirro S, Beeman J W, Capelli S, Pavan M, Previtali E and Gorla P 2006 Scintillating double beta decay bolometers Phys. Atom. Nucl. 69 2109-16

[2] Nagornaya L L et al 2009 IEEE Trans. Nucl. Sci. 56 2513-8

[3] Mikhailik V B, Kraus H, Wahl D, Ehrenberg H and Mykhaylyk M S 2006 Nucl. Instrum. Methods Phys. Res. A 562 513-6

[4] Gironi L et al 2010 Performance of $\mathrm{ZnMoO}_{4}$ crystal as cryogenic scintillating bolometer to search for double beta decay of molybdenum J. Instrum. JINST 5 P11007

[5] Spassky D, Vasil'ev A, Kamenskikh I, Kolobanov V, Mikhailin V, Savon A, Ivleva L, Voronina I and Berezovskaya L 2009 Phys. Status Solidi a 206 1579-83

[6] Nikolaenko T N, Hizhnyi Yu A and Nedilko S G 2005 SCINT2005: Proc. 8th Int. Conf. on Inorganic Scintillators and Their Use in Scientific and Industrial Applications (Alushta, Sept. 2005) ed A Gektin and B Grinyov (Kharkov: ISMA) pp 40 
[7] Zhang Y, Holzwarth N A W and Williams R T 1998 Phys. Rev. B 57 12738-50

[8] Abraham Y B, Holzwarth N A W and Williams R T 2000 Phys. Rev. B 62 1733-41

[9] Abraham Y B, Holzwarth N A W, Williams R T and Matthews E 2001 Phys. Rev. B 64245109

[10] Williams R T, Zhang Y C, Abraham Y and Holzwarth N A W 1999 SCINT99: Proc. 5th Int. Conf. on Inorganic Scintillators and Their Use in Scientific and Industrial Applications (Moscow, Aug. 1999) ed V Mikhailin (Moscow: Yanus-K) pp 118

[11] Hizhnyi Yu A, Nedilko S G and Bilyi M U 2002 Radiat. Eff. Defects Solids 157839

[12] Hizhnyi Yu A, Nedilko S G and Nikolaenko T N 2005 Nucl. Instrum. Methods Phys. Res. A 537 36-9

[13] Nedilko S G, Hizhnyi Yu A and Nikolaenko T N 2005 Phys. Status Solidi c 2 481-4

[14] Nikolaenko T N, Hizhnyi Yu A and Nedilko S G 2008 J. Lumin. 128 807-10

[15] Fujita M, Itoh M, Katagiri T, Iri D, Kitaura M and Mikhailik V B 2008 Phys. Rev. B 77155118

[16] Itoh M, Fujita N and Inabe Yo 2006 J. Phys. Soc. Japan 75084705

[17] Kalinko A, Kuzmin A and Evarestov R A 2009 Solid State Commun. 149 425-8

[18] Reichelt W, Weber T, Soehnel T and Daebritz S 2000 Z. Anorg. Allg. Chem. 626 2020-7

[19] Abrahams S C 1967 J. Chem. Phys. 46 2052-63

[20] Blaha P, Schwarz K, Madsen G, Kvasnicka D and Luitz J 2001 WIEN2k, An Augmented Plane Wave Local Orbitals Program for Calculating Crystal Properties, Karlheinz Schwarz (Austria: Techn. Universitadt Wien) ISBN 3-9501031-1-2

[21] Perdew J P and Wang Y 1992 Phys. Rev. B 45 13244-9

[22] Blochl P E, Jepsen O and Andersen O K 1994 Phys. Rev. B 49 16223-33

[23] Ivleva L, Voronina I, Berezovskaya L, Lykov P, Osiko V and Ishakova L 2008 Crystallogr. Rep. 5 1122-5

[24] Zimmerer G 2007 Radiat. Meas. 42 859-64

[25] Kebabcioglu R and Muller A 1970 Chem. Phys. Lett. 8 59-62

[26] Jitsuhiro S, Nakai H, Hada M and Nakatsuji H 1994 J. Chem. Phys. 101 1029-36

[27] Sobolev V V and Nemoshkalenko V V 1992 Electronic Structure of Solids in the Region of Fundamental Absorption Edge (Kiev: Naukova Dumka)
[28] Ambrosh-Draxl C and Sofo J 2006 Comput. Phys. Commun. $1751-14$

[29] Landau L D and Lifshits E M 1959 Electrodynamics of Continuous Media (Moscow: FizMatGiz)

[30] Mikhailin V V and Vasil'ev A N 1987 Introduction to Solid-State Spectroscopy (Moscow: Izd. MGU)

[31] Nikl M, Bohacek P, Mihokova E, Kobayashi M, Ishii M, Usuki Y, Babin V, Stolovich A, Zazubovich S and Bacci M 2000 J. Lumin. 87 1136-9

[32] Mikhailik V B, Kraus H, Itoh M, Iri D and Uchida M 2005 J. Phys.: Condens. Matter 17 7209-18

[33] Belskiy A N, Kamenskikh I A, Mikhailin V V, Shpinkov I N and Vasil'ev A N 1990 Phys. Scr. 41 530-6

[34] Kamenskikh I A, Mikhailin V V, Shpinkov I N and Vasil'ev A N 2001 Radiat. Eff. Defects Solids 155 153-7

[35] Dujardin C, Pedrini C, Gacon J C, Petrosyan A G, Belsky A N and Vasil'ev A N 1997 J. Phys.: Condens. Matter 9 5229-43

[36] Song K S and Williams R T 1996 Self-trapped Excitons (Berlin: Springer)

[37] Kolobanov V N, Kamenskikh I A, Mikhailin V V, Shpinkov I N, Spassky D A, Zadneprovsky B I, Potkin L I and Zimmerer G 2002 Nucl. Instrum. Methods A 486 496-503

[38] Fujita M, Itoh M, Mitani H, Sangeeta and Tyagi M 2010 Phys. Status Solidi b 247 405-10

[39] Nagirnyi V, Kirm M, Kotlov A, Lushchik A and Jönsson L 2003 J. Lumin. 102/103 597-603

[40] Nagirnyi V, Feldbach E, Jonsson L, Kirm M, Kotlov A, Lushchik A, Nefedov V A and Zadneprovski B I 2002 Nucl. Insturm. Methods Phys. Res. A 486 395-8

[41] Fujita M, Itoh M, Horimoto M and Yokota H 2002 Phys. Rev. B 65195105

[42] Kamenskikh I A, Kirm M, Kolobanov V N, Mikhailin V V, Orekhanov P A, Shpinkov I N, Spassky D A, Vasil'ev A N and Zimmerer G 2001 Radiat. Eff. Defects Solids 154 307-11

[43] Mishra K C, DeBoer B G, Schmidt P C, Osterloh I, Stephan M, Eyert V and Johnson K H 1998 Ber. Bunsenges. Phys. Chem. 102 1772-82

[44] Spassky D, Ivanov S, Kitaeva I, Kolobanov V, Mikhailin V, Ivleva L and Voronina I 2005 Phys. Status Solidi c 2 65-8 